\title{
Erratum to: Role of sirtuins in chronic obstructive pulmonary disease
}

\section{Pusoon Chun}

Erratum to: Arch. Pharm. Res. (2015) 38:1-10

DOI 10.1007/s12272-014-0494-2

Unfortunately, there are a few errors in the published review article titled "Role of sirtuins in chronic obstructive pulmonary disease," DOI 10.1007/s12272-014-0494-2. These errors should be corrected as follows:
In Table 1, Deacytylase should be corrected as Deacetylase. Desuccinlylase should be corrected as Desuccinylase.

In Acknowledgements, "This work was supported by the 2012 Inje University research grant" should be corrected as "This work was supported by the 2013 Inje University research grant."

The online version of the original article can be found under doi:10.1007/s12272-014-0494-2.

P. Chun $(\bowtie)$

College of Pharmacy, Inje University, 197 Inje-ro, Gimhae,

Gyeongnam 621-749, Korea

e-mail: pusoon@inje.ac.kr 